\title{
Choroidal Congestion after Trabeculectomy
}

\author{
Maria Marenco ${ }^{a, b} \quad$ Luigi Borgia $^{c} \quad$ Aldo Vagge ${ }^{a, b} \quad$ Michele lester $^{a, b}$ \\ ${ }^{a}$ Clinica Oculistica, DiNOGMI, University of Genoa, Genoa, Italy; bIRCCS Ospedale \\ Policinico San Martino, Genoa, Italy; 'Casa di Cura "Clinica Montallegro", Genoa, Italy
}

\section{Keywords}

Trabeculectomy · Spectral domain optical coherence tomography Choroidal congestion

\begin{abstract}
The aim of this study is to report a case of choroidal congestion after trabeculectomy. This is a retrospective case report. An 85-year-old male with advanced open-angle glaucoma underwent trabeculectomy in both eyes and during follow-up he complained of visual impairment in the right eye. Thus, spectral domain optical coherence tomography (OCT) was performed and showed choroidal congestion in one eye 1 month after surgery together with a decrease of visual acuity. Symptoms and exam findings resolved with systemic steroids after a 6-month follow-up. OCT is essential for detecting choroidal congestion. This finding can be a possible complication after trabeculectomy and can benefit from steroid treatment.
\end{abstract}

(C) 2019 The Author(s)

Published by S. Karger AG, Basel

\section{Introduction}

Open-angle glaucomas are chronic, progressive optic neuropathies, resulting in loss of retinal fiber layer leading to visual field loss and visual impairment [1]. Since elevated intraocular pressure (IOP) is an important risk factor for glaucoma progression, treatments are directed to IOP reduction [2,3]. Trabeculectomy is an effective surgical method for reducing 
IOP, but it is associated with postoperative complications that can also affect the choroid such as choroidal effusion and/or hemorrhage [4].

Modern glaucoma surgery has minimized the incidence of postoperative hypotony and resultant choroidal effusion, but it is still prevalent. Comparison studies on glaucoma surgery showed early postoperative choroidal effusion rates from $15 \%$ [5] to $10 \%$ [6].

The choroid is the posterior portion of the uveal tract and nourishes the outer portion of the retina. The choriocapillaris is the largest capillary bed with the highest rates of blood flow in the body and can accommodate throughput of multiple red blood cells at once [7]. Its vasculature derives from the ophthalmic artery and is composed of the choriocapillaris layer, the medium vessel layer, and the outer layer of large vessels. Blood is discharged from the lobules of the choriocapillaris by collecting venules that join the afferent veins. Veins of larger lumen form the subcapillaris plexus and flow into the vortex veins. The vortex veins join the orbital venous system after leaving the eye [8]. The wall of the choriocapillaris has large fenestrations that allow proteins to escape and bring with it passive diffusion of fluid into the suprachoroidal space. This fluid is prevented from entering the subretinal space by the zonula occludens found in the retinal pigment epithelium. The IOP exerts a force to prevent too much fluid from accumulating in the suprachoroidal space to counteract the hydrostatic force of fluid following the proteins exiting the large fenestrations of the choriocapillaris [7-10].

In this case report we show choroidal venous congestion after trabeculectomy by using optical coherence tomography (OCT).

\section{Case Report}

An 85-year-old man was referred to our department in October 2015 with a diagnosis of advanced open-angle glaucoma in both eyes for uncontrolled IOP despite medicines and previous laser treatment.

His ocular history included elevated myopia and epiretinal membrane in both eyes, not clinically significant in the right eye, while significant in the left eye with a foveal schisis. He had phacoemulsification in the right eye (September 2006), Yag capsulotomy in the right eye (December 2008), and argon laser trabeculoplasty in the right eye (May 2015).

Medical history was significant for an autoimmune lung disease under systemic steroid treatment (prednisone $25 \mathrm{mg} /$ day).

The patient's best corrected visual acuity (BCVA) was 20/22-20/25 in the right eye with a myopic correction $\left(-3.50\right.$ to $\left.0.75 / 105^{\circ}\right)$ and $20 / 200$ weak in the left eye with a myopic correction $(-14.00$ spherical D).

He was under treatment with lowering topical medications (timolol, brimonidine, latanoprost). His IOP was $19 \mathrm{~mm} \mathrm{Hg}$ in the right eye and $22 \mathrm{~mm} \mathrm{Hg}$ in the left eye.

Since his visual field loss was rapidly progressing in both eyes, we decided to perform trabeculectomy, first in the left (November 2015) and then in the right eye (February 2016).

Following filtering surgery, the IOP was well controlled without drops in the left eye, while the right eye required repeated ocular massages during the first 2 weeks of follow-up until reaching a stable IOP of $5 \mathrm{~mm} \mathrm{Hg}$ [11].

After trabeculectomy, there was no change in BCVA in the left, while in the right eye it decreased to 20/66 without recovering during the first months of follow-up. Consequently, spectral domain OCT was performed using the Heidelberg Spectralis (Heidelberg retina angiography + OCT) (Heidelberg Engineering, Heidelberg, Germany) and showed choroidal venous congestion in the right eye (Fig. 1). Thus, the patient underwent systemic steroid 
(prednisone $1 \mathrm{mg} / \mathrm{kg} /$ day) slowly tapered in 2 months until usual treatment of $25 \mathrm{mg} /$ day. Choroidal congestion progressively decreased until disappearing in June 2016 (Fig. 2, 3). At the last follow-up visit in December 2018, BCVA was still 20/28, IOP was $12 \mathrm{~mm} \mathrm{Hg}$ without drops, and OCT showed the same aspect of choroid as it had before surgery (Fig. 4, 5).

\section{Discussion}

Glaucoma treatment is related to a decrease of IOP, first with medical treatment, then with laser or surgical approach [1]. The decrease of IOP has been shown to decrease the rate of progression; however, the target IOP is not easy to find for each patient. Based on the stage of the disease or on the additional risk factors, target IOP can change significantly. When a relatively too high IOP is surgically obtained, this value cannot change the rate of progression of the disease, while on the other hand relatively too low IOP could create a clinically significant ocular hypotony.

The hydrostatic pressure gradient of the suprachoroidal space is created by the IOP, while the osmotic pressure is created by the protein and subsequent fluid diffusion through the choriocapillaris. The difference between these pressures allows the suprachoroidal space to be larger or a true space versus a smaller or potential space [9]. Evidence shows that a higher IOP creates a greater pressure difference with the hydrostatic pressure of the choroid, while a lower IOP creates a smaller pressure difference [8]. Once the IOP is lower than the episcleral venous pressure, and if there is a large inflammatory response and subsequent excessive protein leakage from the choriocapillaris, then a choroidal effusion can occur [12]. It should be noted that many patients have IOP lower than the episcleral venous pressure but do not produce suprachoroidal effusions, presumably because there is no inflammation that causes leakage of large proteins from choroidal vessels. In contrast, hemorrhagic choroidal effusions occur due to rupture of the posterior ciliary vessels that bridge the suprachoroidal space from the sclera to the choroid; a Valsalva maneuver can facilitate this complication [9].

Serous choroidal effusions can be relatively painless and accumulate slowly over the course of days or weeks after surgery [10]. These may range from being entirely asymptomatic to causing a significant degree of vision loss by involving the visual axis or by anteriorly shifting the position of the lens-iris diaphragm. In contrast, a hemorrhagic choroidal effusion, also known as a suprachoroidal hemorrhage, is characterized by a sudden, often very painful accumulation of blood in the suprachoroidal space caused by disruption of the posterior ciliary arteries [13].

If ocular hypotony is just for a short period, most of the time patients do not have any visual problem, but when it is for a long period, hypotonic maculopathy may occur changing forever the capacity of the patient to see. When the hypotony is just after surgery, the eye is usually normal, but in some cases it is possible to have choroidal effusion.

A choroidal effusion, hypotony, and development of a shallow anterior chamber were also described in a patient following a transconjunctival needling revision of an encapsulated filtering bleb, outlining the importance of decreasing IOP slowly [14].

In our patient, a possible additional cause of choroidal congestion could be an unstable IOP during the first 2 weeks of follow-up, during which IOP decreased rapidly by nearly 10 $\mathrm{mm} \mathrm{Hg}$ after each ocular massage [11]. This idea would be supported by the absence of choroidal alterations in the other eye postsurgery, probably due to a stable IOP following trabeculectomy and the normal choroidal thickness years after surgery. In this case a visual acuity reduction without any clinical signs was the reason of the OCT evaluation, which was 
fundamental in detecting choroidal modifications and to start the steroid treatment, instead of waiting for a possible choroidal detachment.

In conclusion, in glaucoma patients, choroidal effusion is mostly due to changes of differential pressure between the inside of the eyes and the inside of the vessels together with proinflammatory conditions. The balance between IOP and systemic pressure at eye level changes after surgery - the vessel pressure is relatively higher than IOP. In some patients this change can cause choroidal effusion. This difference in differential pressure could first create an increase in the vessel diameter of the choroid, causing a thickening of the choroid. Choroidal congestion could be a possible finding following trabeculectomy and could be treated with systemic steroids. Further studies are needed to elucidate choroidal behavior after trabeculectomy.

\section{Statement of Ethics}

The subject gave his informed consent. This paper is a retrospective case report and as such does not need to be approved by the institute's committee on human research.

\section{Disclosure Statement}

This research received no specific grant from any funding agency in the public, commercial, or not-for-profit sectors. None of the authors has any proprietary interest in the development or marketing of any products mentioned in this paper.

\section{Funding Sources}

The authors have no funding to declare.

\section{Author Contributions}

M.I.: surgeon. M.I. and L.B.: development of the project. M.I., L.B., M.M., and A.V.: writing of the manuscript.

\section{References}

1 European Glaucoma Society Terminology and Guidelines for Glaucoma. 4th Edition - Chapter 2:

Classification and terminology Supported by the EGS Foundation: Part 1: Foreword; Introduction; Glossary;

Chapter 2 Classification and Terminology. Br J Ophthalmol 2017;101(5):73-127.

2 The AGIS Investigators. The Advanced Glaucoma Intervention Study (AGIS): 7. The relationship between control of intraocular pressure and visual field deterioration. Am J Ophthalmol. 2000 Oct;130(4):429-40.

3 Musch DC, Gillespie BW, Lichter PR, Niziol LM, Janz NK; CIGTS Study Investigators. Visual field progression in the Collaborative Initial Glaucoma Treatment Study the impact of treatment and other baseline factors. Ophthalmology. 2009 Feb;116(2):200-7.

4 Rulli E, Biagioli E, Riva I, Gambirasio G, De Simone I, Floriani I, et al. Efficacy and safety of trabeculectomy vs nonpenetrating surgical procedures: a systematic review and meta-analysis. JAMA Ophthalmol. 2013 Dec;131(12):1573-82. 


\section{Case Reports in Ophthalmology}

5 Budenz DL, Barton K, Feuer WJ, Schiffman J, Costa VP, Godfrey DG, et al.; Ahmed Baerveldt Comparison Study Group. Treatment outcomes in the Ahmed Baerveldt Comparison Study after 1 year of follow-up. Ophthalmology. 2011 Mar;118(3):443-52.

6 Gedde SJ, Herndon LW, Brandt JD, Budenz DL, Feuer WJ, Schiffman JC; Tube Versus Trabeculectomy Study Group. Postoperative complications in the Tube Versus Trabeculectomy (TVT) study during five years of follow-up. Am J Ophthalmol. 2012 May;153(5):804-814.e1.

7 Agarwal A. Gass' atlas of macular diseases. Volume 1. 5th ed. Amsterdam: Elsevier Saunders; 2012. p. 4.

8 Emi K, Pederson JE, Toris CB. Hydrostatic pressure of the suprachoroidal space. Invest Ophthalmol Vis Sci. 1989 Feb;30(2):233-8.

9 Bakir B, Pasquale LR. Causes and treatment of choroidal effusion after glaucoma surgery. Semin Ophthalmol. 2014 Sep-Nov;29(5-6):409-13.

10 Schrieber C, Liu Y. Choroidal effusions after glaucoma surgery. Curr Opin Ophthalmol. 2015 Mar;26(2): 134-42.

11 Traverso CE, Greenidge KC, Spaeth GL, Wilson RP. Focal pressure: a new method to encourage filtration after trabeculectomy. Ophthalmic Surg. 1984 Jan;15(1):62-5.

12 Sit AJ, McLaren JW. Measurement of episcleral venous pressure. Exp Eye Res. 2011 Sep;93(3):291-8.

13 Chu TG, Green RL. Suprachoroidal hemorrhage. Surv Ophthalmol. 1999 May-Jun;43(6):471-86.

14 Potash SD, Ritch R, Liebmann J. Ocular hypotony and choroidal effusion following bleb needling. Ophthalmic Surg. 1993 Apr;24(4):279-80.

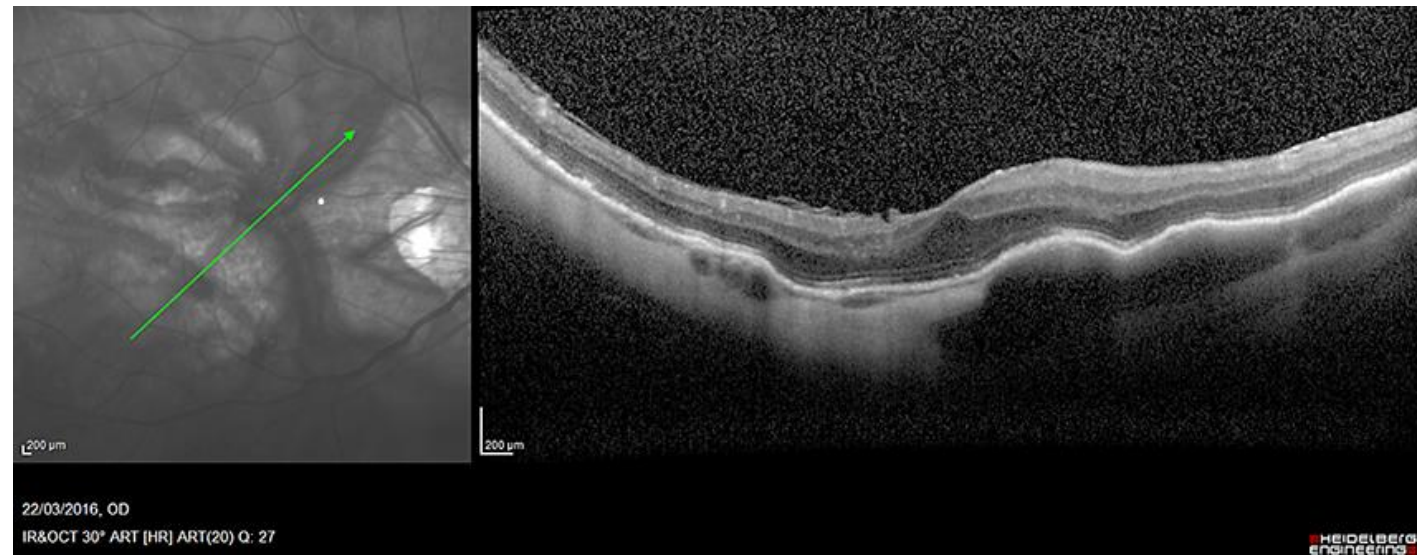

Fig. 1. Choroidal venous congestion in the right eye about 1 month after the filtering surgery. 


\section{Case Reports in Ophthalmology}

\begin{tabular}{l|l}
\hline Case Rep Ophthalmol 2019;10:384-390 \\
\hline DOI: 10.1159/000504318 & $\begin{array}{l}\text { (c) 2019 The Author(s). Published by S. Karger AG, Basel } \\
\text { www.karger.com/cop }\end{array}$ \\
\hline
\end{tabular}

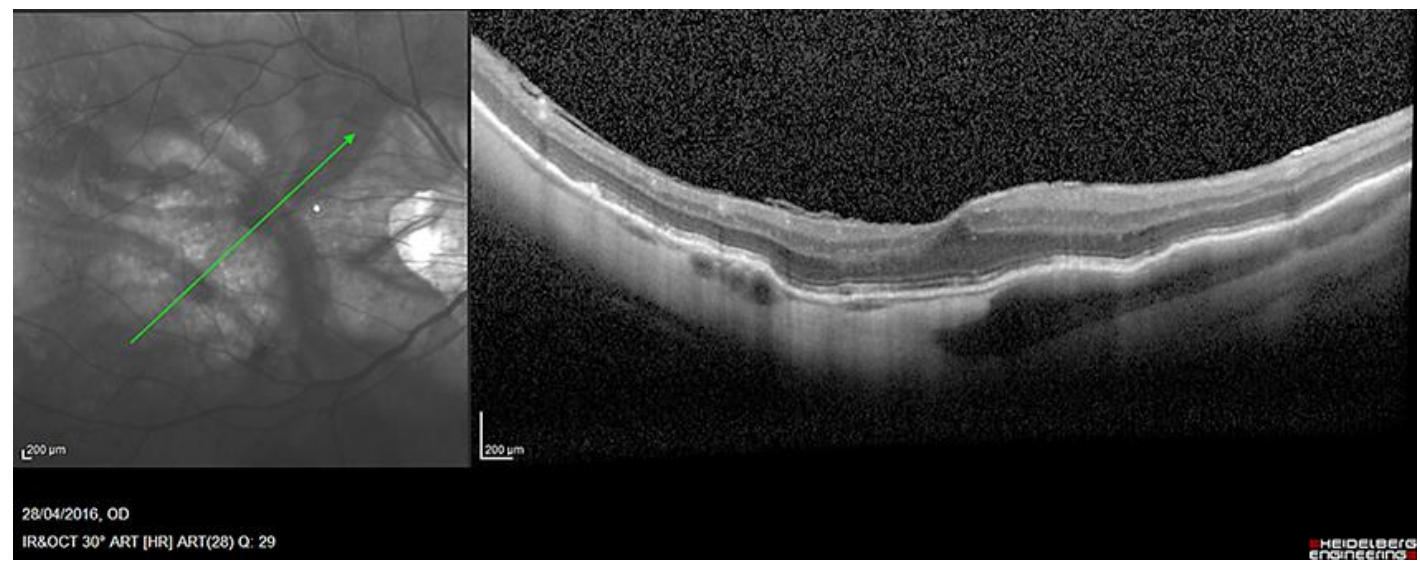

Fig. 2. Reduction of choroidal venous congestion in the right eye 2 months after the filtering surgery.

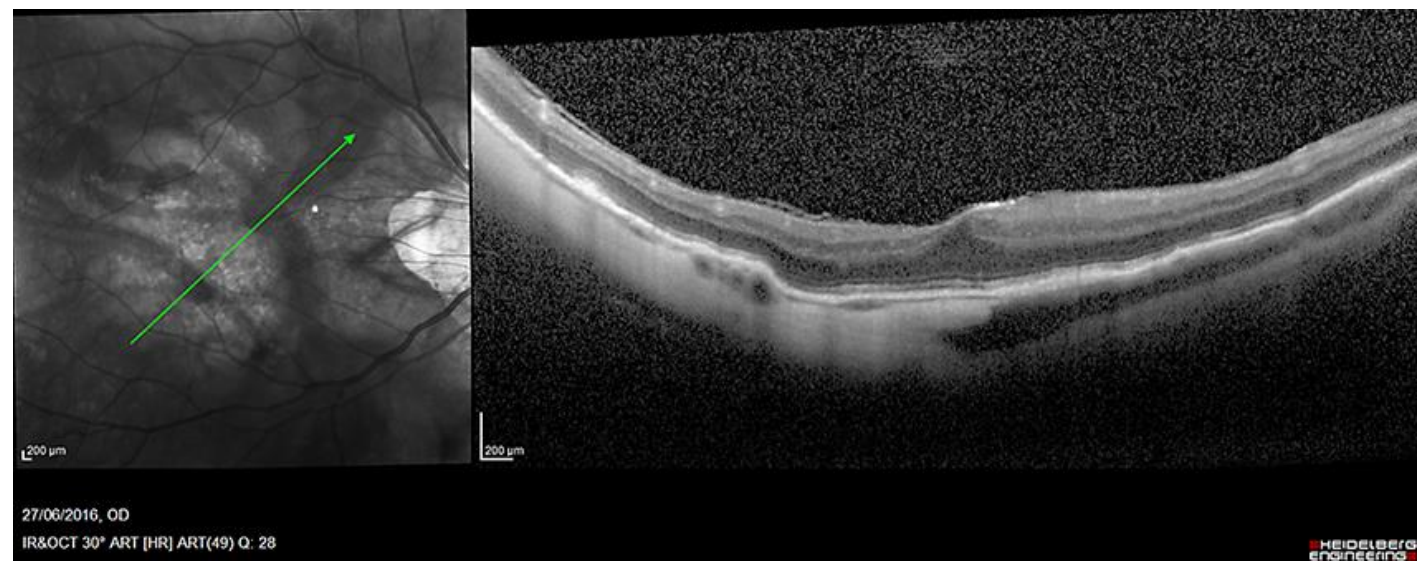

Fig. 3. Reduction of choroidal venous congestion in the right eye 4 months after the filtering surgery.

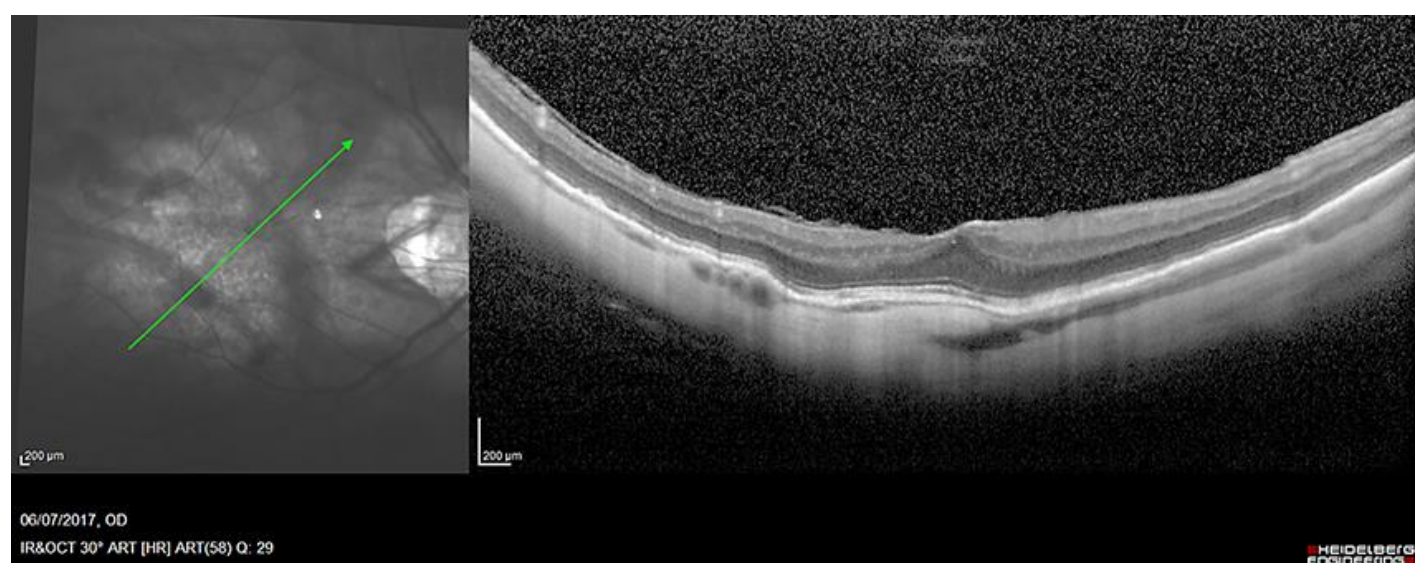

Fig. 4. Choroidal scan shows no more changes in the right eye 1 year after the filtering surgery. 


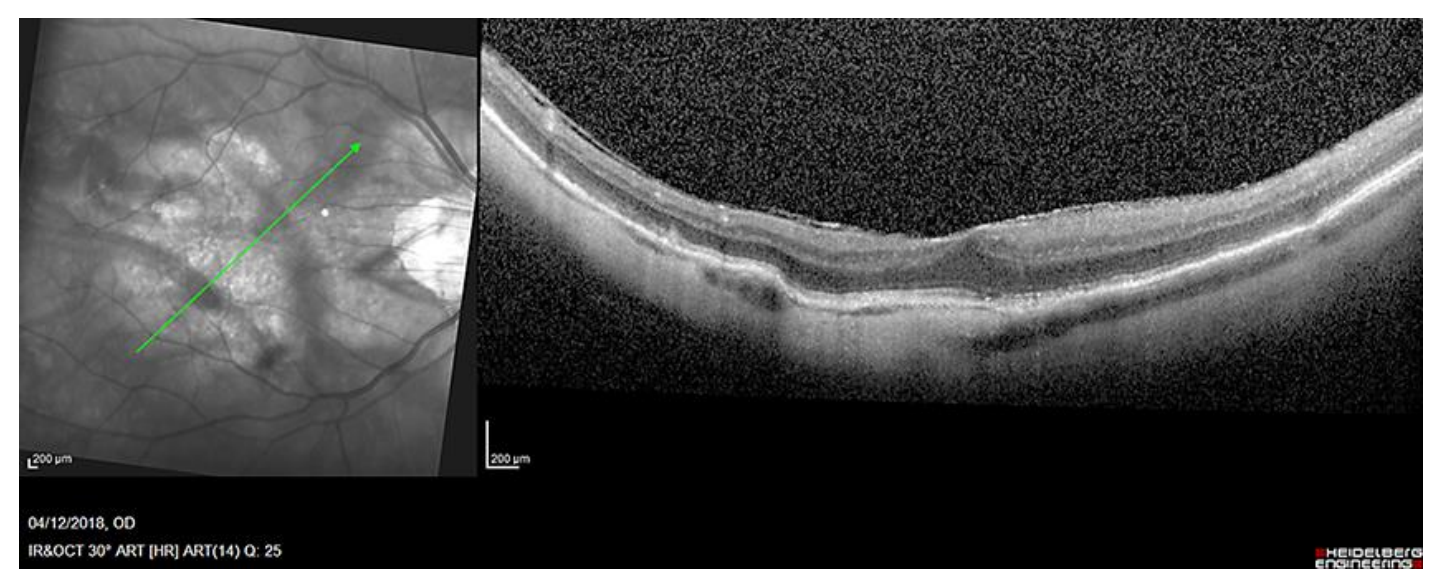

Fig. 5. Choroidal scan shows no more changes in the right eye 2 years after the filtering surgery. 